\title{
Improvement in the implementation of Helicobacter pylori management guidelines among primary care physicians following a targeted educational intervention
}

\section{Doron Boltin $^{\mathrm{a}, \mathrm{b}}$, Iris Dotan ${ }^{\mathrm{a}}$, Shlomo Birkenfeldc}

Rabin Medical Center, Beilinson Campus, Petah Tikva, Israel and the Sackler Faculty of Medicine, Tel Aviv University; Bat Yamon Medical Center, Clalit Health Services, Bat Yam; Tel Aviv and Central Administration, Tel Aviv, Israel

\section{Abstract}

\begin{abstract}
Background Consensus guidelines recommend that in regions with a high rate of clarithromycin resistance, Helicobacter pylori (H. pylori) infection be treated with 4 drugs. Compliance with this recommendation among primary care physicians (PCPs) is low. We aimed to examine whether PCP compliance with $H$. pylori treatment recommendations increased following a targeted educational intervention.
\end{abstract}

Methods A questionnaire assessing H. pylori treatment was sent to $>2000$ PCPs in June 2015 and June 2018. In the interim, 3 interventions were performed: distribution of printed materials, educational outreach visits, and education over a social media platform.

Results A total of 635 PCPs returned questionnaires, including 314 in 2015 and 321 in 2018 (148 [46.3\%] male, age $44.7 \pm 10.9$ years). The number of PCPs who recommended a 4 -drug treatment protocol increased from $12(3.8 \%)$ in 2015 to $119(37.1 \%)$ in $2018(\mathrm{P}<0.001)$. The number of PCPs who recommended bismuth- or levofloxacin-based therapy for second-line treatment increased from $95(30.3 \%)$ in 2015 to $247(77.1 \%)$ in $2018(\mathrm{P}<0.001)$. Independent predictors for a 4-drug treatment protocol included central clinic location (odds ratio [OR] 2.78, 95\% confidence interval $[\mathrm{CI}] 1.38-5.60 ; \mathrm{P}<0.003)$, exposure to printed educational materials (OR $1.64,95 \% \mathrm{CI} 0.99-2.72 ; \mathrm{P}=0.04$ ) and exposure to the social media platform (OR 6.60, 95\%CI 3.08-14.13; $\mathrm{P}<0.001$. There were no independent predictors of compliance with second-line treatment.

Conclusions PCP compliance with H. pylori guidelines remains suboptimal. Educational initiatives may be effective in increasing PCPs' knowledge and compliance with guidelines. Direct web-based interaction between PCPs and gastroenterologists may be particularly effective.

Keywords Helicobacter pylori, treatment, guidelines, primary care physician, social media

Ann Gastroenterol 2019; 32 (1): 1-8
${ }^{a}$ Division of Gastroenterology, Rabin Medical Center, Beilinson Campus, Petah Tikva, Israel and the Sackler Faculty of Medicine, Tel Aviv University, Tel Aviv, Israel (Doron Boltin, Iris Dotan); ${ }^{\mathrm{b} B a t}$ Yamon Medical Center, Clalit Health Services, Bat Yam, Israel (Doron Boltin); 'Tel Aviv and Central Administration, Clalit Health Services, Tel Aviv (Shlomo Birkenfeld), Israel

\section{Conflict of Interest: None}

Correspondence to: Dr. Doron Boltin, MBBS, Division of Gastroenterology, Rabin Medical Center, 39 Jabotinski Street, Petah Tikva, Israel 49100, e-mail: dboltin@gmail.com

Received 9 September 2018; accepted 15 October 2018; published online 24 November 2018

DOI: https://doi.org/10.20524/aog.2018.0329

\section{Introduction}

Helicobacter pylori (H. pylori) infection has a direct impact on public health due to its role in the development of chronic gastritis, peptic ulcer disease (PUD) and gastric cancer. Eradication of the organism may improve the outcomes of these diseases [1]. The optimal regimen for treating $H$. pylori infection varies according to geographic region, since the choice of antibiotics must be tailored according to local resistance data. Failure to account for regional differences in $H$. pylori antibiotic resistance may significantly reduce the efficacy of $H$. pylori eradication regimens [2]. Therefore, ineffective eradication regimens not only fail to reduce the burden of $H$. pylori infection and associated pathologies, but also introduce secondary public health concerns, such as selection of antibiotic- 
resistant $H$. pylori strains, increased morbidity related to repeated antibiotic exposure, and increased costs. Current guidelines, including the Maastricht V/Florence Consensus Report, the American College of Gastroenterology Clinical Guideline and the Toronto Consensus, recommend adding a nitroimidazole drug to traditional clarithromycin (CLA)-based triple therapy (TT) for $H$. pylori eradication among treatmentnaïve subjects, in regions with a high rate of CLA resistance [24]. Nevertheless, the implementation of this recommendation is suboptimal. In a study performed by our group in 2015 we found that over $93 \%$ of primary care physicians (PCPs) continued to prescribe TT despite the fact that our region has a high rate of primary CLA resistance [5-7]. Furthermore, only $30 \%$ of PCPs provided a second-line eradication regimen compatible with current guidelines. Additional gaps were identified regarding the indications for $H$. pylori testing and attitudes towards the oncogenic potential of the organism.

It has previously been shown that educational outreach visits (EOV) involving face-to-face meetings with healthcare professionals lead to changes in prescribing practices [8]. Such visits are more effective in changing PCP behavior than are alternative methods, such as audit with feedback. Likewise, the distribution of printed educational materials may have a beneficial effect on professional practice outcomes [9]. Over the past decade, electronic consultation (e-consult) has become an increasingly popular tool to facilitate provider-to-provider communication and may positively impact decision making [10-12]. Social media platforms also allow effective collaboration between healthcare professionals and have been shown to enhance educational interactions between health providers [13-15].

Following publication of the Maastricht/Florence V consensus report we advanced a national educational initiative to address the gaps identified in our previous study [5]. The aim of the present study was to assess the change in $H$. pylori-treatment practices following the implementation of a targeted educational intervention and to assess the relative effectiveness of each initiative.

\section{Materials and methods}

\section{Intervention}

Following the publication of our previous survey [5] three interventions were performed. Firstly, printed materials were distributed to PCPs at a national level. These included patient education flyers regarding the implications of $H$. pylori infection and treatment protocols, a letter addressed to PCPs summarizing the key statements of the Maastricht/Florence V Consensus Report [2], and how-to-prescribe cards. Secondly, EOVs were performed by trained personnel, during which the contents of the printed materials were conveyed orally. Thirdly, the key statements of the Maastricht/Florence V Consensus Report were transferred over a preexisting social media platform. This social media platform is a forum through which PCPs can interact with a single consultant gastroenterologist. The platform functions as a message board, on which all participants can view all of the comments posted, and may be accessed from both desktop and mobile devices.
The consultant gastroenterologist typically responds to questions posed by PCPs within $1 \mathrm{~h}$, and often in real-time.

\section{Questionnaire}

The questionnaire previously sent in June 2015 was redistributed in June 2018 with the addition of one question (Question 14) which assessed attitudes towards testing H. pylori prior to commencing long-term aspirin or nonsteroidal anti-inflammatory drug (NSAID) therapy in patients with a history of PUD. This question was included to differentiate adherence to the Mastricht V/Florence Consensus Report, which recommends $H$. pylori testing prior to aspirin and NSAID therapy in subjects with a history of PUD, from adherence to the ACG Clinical Guideline, which mandates testing prior to aspirin and NSAID therapy, regardless of any history of PUD [3].

The final questionnaire consisted of 14 multiple choice questions, including 2 related to treatment, 5 related to $H$. pylori detection, 3 related to gastric cancer and 4 related to screening. Questions were jointly written by an expert panel of 3 consultant gastroenterologists, including one contributor to the Maastricht IV and Maastricht V/Florence Consensus Report. The panel sought to address topics within the realm of general practice yet are presumed to be poorly managed. Study questions with answer options are shown in Supplementary Table 1. Demographic variables were collected, including age, sex, clinic location and estimated number of $H$. pylori diagnosis or treatment encounters per month. In addition, subjects were asked about exposure to printed materials or EOVs during the preceding 3 months. PCP participation in the social media platform was correlated with electronic data from the system administrator.

\section{Distribution}

Questionnaires were distributed by e-mail to the personal accounts of approximately 2000 PCPs working for Clalit Health Services (CHS) in Israel. CHS is the largest health maintenance organization in Israel and the second largest health maintenance organization in the world, with a staff of 34,000 employees and more than 3.8 million enrollees. Healthcare is delivered through a network of facilities that include 14 hospitals, 1300 primary and specialized clinics, and a network of pharmacies and dental clinics throughout 8 districts.

Responses were collected electronically during a period of 14 days, after which no further responses were allowed. PCPs who responded to $<90 \%$ of questions were excluded. Only one response per IP address was permitted.

\section{Endpoints}

The primary outcome measure assessed was the change in the proportion of PCPs who provided first- and second-line therapy consistent with current guidelines, as defined below. Secondary measures included changes in PCP attitudes with respect to $H$. pylori detection, malignant potential and screening. 


\section{Statistical analysis}

Given the PCP population size of 2000, we calculated that a sample size of 322 would be necessary to allow a margin of error of $5 \%$ with $95 \%$ confidence. Data analysis was performed using SAS 9.4 statistical analysis software (SAS Institute Inc. NC, USA). Continuous variables were reported as mean \pm SD. Normality of distribution of continuous variables was assessed using the Kolmogorov-Smirnov test (cutoff at $\mathrm{P}=0.01$ ). Categorical variables were described using frequency distributions and were presented as frequencies: $\mathrm{n}(\%)$. Depending on the distribution, continuous variables were compared across groups using oneway analysis of variance (ANOVA) or the Kruskal-Wallis test. Pairwise, post hoc comparisons for significance across differences were assessed by Bonferroni's test or the Mann-Whitney $U$ test. Categorical variables were compared across groups using the chi-square test (exact as necessary). Pearson's correlation coefficient was used to test for correlations between variables. A multivariate regression model to assess for predictors compliance with first- and second-line treatment included the following variables: sex, clinic location, volume of $H$. pylori patients and educational interventions. The dependent variable of compliance with guidelines was defined as any 4-drug regimen for first-line therapy, and bismuth-based quadruple (BQT) or levofloxacin-based therapy for second-line eradication. PCP respondents who chose to refer patients to a gastroenterologist for second-line treatment were omitted from the model. All tests were 2 -sided and considered significant at $\mathrm{P}<0.05$.

\section{Results}

\section{Respondents}

A total of 332 PCPs returned questionnaires. Eleven respondents completed less than $90 \%$ of the survey and were excluded. Therefore, 321 respondents (age $44.7 \pm 10.9$ years) were included in the final data set, including 148 (46.3\%) males. The characteristics of respondents are shown in Table 1. Two hundred seventy-seven PCPs $(86.3 \%)$ reported a low number $(<10)$ of H. pylori encounters per month. Exposure to $\geq 1$ educational initiative was reported by 208 (64.8\%) PCPs, including 98 (30.5\%) exposed to EOVs, 150 (46.7\%) exposed to printed materials and $48(15 \%)$ who accessed the social media platform.

\section{H. pylori treatment}

The most commonly recommended first-line treatment was standard TT for 7-10 days in 2015 (248 [80.0\%]) and TT for 14 days in 2018 (115 [35.8\%]) $(\mathrm{P}<0.001)$ (Table 2). The number of PCPs who recommended a 4-drug treatment protocol increased from $12(3.8 \%)$ in 2015 to 119 (37.1\%) in $2018(\mathrm{P}<0.001)$ (Fig. 1). The most common 4-drug regimen was sequential therapy in $2015(11$ [3.5\%]) and concomitant therapy in 2018 (91 [28.3\%]). The most commonly recommended second-line treatment was metronidazole-based
Table 1 Characteristics of respondents, 2018

\begin{tabular}{lc}
\hline Characteristics & $\mathrm{N}(\%)$ \\
\hline Total & $321(100)$ \\
\hline Male sex & $148(46.3)$ \\
Age, years, mean (SD) & $44.7(10.9)$ \\
\hline Hp patients/month & \\
$<10$ & $277(86.3)$ \\
$10-30$ & $17(5.3)$ \\
$31-50$ & $12(3.7)$ \\
$>50$ & $15(4.7)$ \\
Clinic location & \\
Tel Aviv (central) region & $69(21.5)$ \\
Jerusalem & $25(7.8)$ \\
Mid-north(Sharon) & $48(15.0)$ \\
North (Galilee/Golan) & $110(34.6)$ \\
Mid-south (Shefela) & $26(8.1)$ \\
South (Negev) & $41(12.8)$ \\
\hline Exposure to educational outreach visits & $98(30.5)$ \\
Exposure to printed materials & $150(46.7)$ \\
Interaction with GI consult over social media platform & $48(15.0)$ \\
\hline Hp, Helicobacter pylori; SD, standard deviation; GI, gastroenterology
\end{tabular}

TT in 2015 (122 [38.9\%]) and BQT in 2018 (199 [62.0\%]). The number of PCPs who recommended bismuth- or levofloxacinbased therapy for second-line treatment increased from $95(30.3 \%)$ in 2015 to $247(77.1 \%)$ in $2018(\mathrm{P}<0.001)$.

\section{Change in attitudes towards H. pylori 2015-2018}

In 2018 more PCPs recommended treatment for $H$. pylori, regardless of symptoms, and recommended confirming eradication with a noninvasive test (130 [41.4\%] vs. 186 [57.9\%] $(\mathrm{P}<0.001)$ and 137 [43.3\%] vs. 178 [55.5\%] $(\mathrm{P}=0.021)$ in 2015 vs. 2018, respectively) (Table 3 ). The number of PCPs who acknowledged a definite association between $H$. pylori and gastric cancer, and agreed that treatment reduces cancer risk, also increased $(130$ [41.1\%] vs. 193 [60.1\%] $(\mathrm{P}<0.001)$ and 188 [65.1\%] vs. 254 [79.4\%] $\mathrm{P}<0.001)$ in 2015 vs. 2018 , respectively. There was no significant change in the number of PCPs who "usually" or "consistently" screened first-degree relatives of gastric cancer patients (87 [30.9\%] vs. 112 [35.2\%] in 2015 vs. 2018 , respectively [P=0.26]). The number of PCPs who "usually" or "consistently" recommended screening prior to commencing long-term NSAID therapy remained low (39 [14.1\%] vs. 46 [14.4\%] in 2015 vs. 2018 , respectively [ $\mathrm{P} \geq 0.99$ ]). Among PCPs who did not "usually" or "consistently" screen prior to NSAID therapy, 79 (29.0\%) would nevertheless "usually" or "consistently" screen prior to NSAID therapy if the patient had a history of PUD. 
4 D. Boltin et al

Table 2 First- and second-line $H p$ treatment recommended by PCPs in 2015 and 2018

\begin{tabular}{|c|c|c|c|c|}
\hline \multirow[t]{2}{*}{$\mathrm{Qu}$} & & 2015 & 2018 & $\mathrm{P}$ \\
\hline & Total, N (\%) & $314(100)$ & $321(100)$ & \\
\hline \multirow[t]{8}{*}{1} & First-line treatment & & & \\
\hline & 3-drug protocol & $292(93.0)$ & $202(62.9)$ & $<0.001$ \\
\hline & Clarithromycin triple $7-10$ days & $248(80.0)$ & $87(27.1)$ & \\
\hline & Clarithromycin triple 14 days & $44(14.0)$ & $115(35.8)$ & \\
\hline & 4-drug protocol & $12(3.8)$ & $119(37.1)$ & $<0.001$ \\
\hline & Sequential therapy & $11(3.5)$ & $19(5.9)$ & \\
\hline & Concomitant therapy & $1(0.3)$ & $91(28.3)$ & \\
\hline & Bismuth-based quadruple & $0(0.0)$ & $9(2.8)$ & \\
\hline \multirow[t]{10}{*}{2} & Second-line treatment & & & \\
\hline & Consistent with guidelines & $95(30.3)$ & $247(77.1)$ & $<0.001$ \\
\hline & Bismuth-based quadruple & $85(27.1)$ & $199(62.0)$ & \\
\hline & Levofloxacin-based triple & $10(3.2)$ & $48(15.1)$ & \\
\hline & Inconsistent with guidelines & $144(45.9)$ & $20(6.2)$ & $<0.001$ \\
\hline & Repeat first-line treatment & $21(6.7)$ & $19(5.9)$ & \\
\hline & Nitroimidazole-based triple & $122(38.9)$ & $0(0)$ & \\
\hline & No treatment & $1(0.3)$ & $1(0.3)$ & \\
\hline & Other & & & \\
\hline & GI referral & $75(23.9)$ & $54(16.8)$ & \\
\hline
\end{tabular}

Hp, Helicobacter pylori; GI, gastroenterology

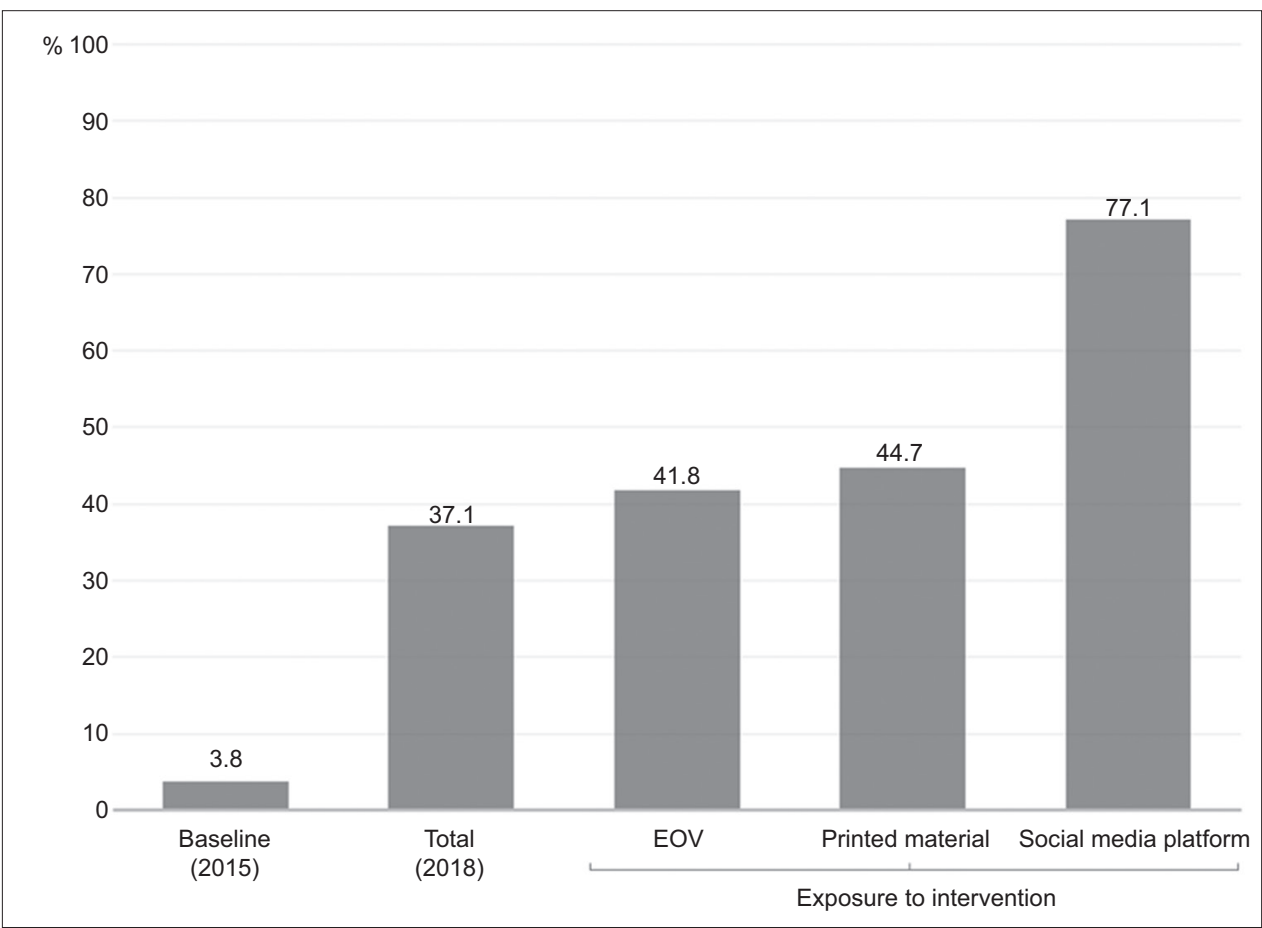

Figure 1 Compliance with guidelines for first-line Helicobacter pylori treatment at baseline (2015) and following exposure to educational interventions $(2018)^{*}$

${ }^{*}$ Compliance defined as recommendation for 4-drug regimen

EOV, educational outreach visit 
Table 3 Attitudes towards Hp detection, malignant potential and screening among PCPs in 2015 and 2018

\begin{tabular}{|c|c|c|c|c|}
\hline $\mathrm{Qu}$ & & 2015 & 2018 & $\mathrm{P}$ \\
\hline & Detection of H. pylori & & & \\
\hline \multirow[t]{5}{*}{3} & What is your preferred noninvasive test to diagnose H. pylori infection? & & & \\
\hline & ${ }^{13} \mathrm{C}$-urea breath test & $198(63.1)$ & $252(78.5)$ & 0.02 \\
\hline & Stool antigen test & $80(25.5)$ & $67(20.9)$ & \\
\hline & Serology & $1(0.3)$ & $1(0.3)$ & \\
\hline & None & $7(2.2)$ & $1(0.3)$ & \\
\hline \multirow[t]{5}{*}{4} & How many days do you withhold PPIs and antibiotics prior to H. pylori testing? & & & \\
\hline & 0 & $4(1.3)$ & $3(0.9)$ & $<.001$ \\
\hline & $5-7$ & $165(52.5)$ & $116(36.1)$ & \\
\hline & 14 & $99(31.5)$ & $175(54.5)$ & \\
\hline & 30 & $18(5.7)$ & $26(8.1)$ & \\
\hline \multirow[t]{5}{*}{5} & Do you confirm $H$. pylori eradication with a noninvasive test? & & & \\
\hline & Yes, in all patients & $137(43.6)$ & $178(55.5)$ & 0.02 \\
\hline & Only if symptomatic & $108(34.4)$ & $88(27.4)$ & \\
\hline & Only if PUD or malignancy & $20(6.4)$ & $18(5.6)$ & \\
\hline & Never & $49(15.6)$ & $35(10.9)$ & \\
\hline \multirow[t]{3}{*}{6} & What do you do when confronted with a positive H. pylori test in an asymptomatic patient? & & & \\
\hline & Treat regardless of symptoms & $130(41.4)$ & $186(57.9)$ & $<0.001$ \\
\hline & Treat only if symptomatic & $182(57.9)$ & $132(41.1)$ & \\
\hline \multirow[t]{2}{*}{7} & Do you seek H. pylori in patients with symptoms suggestive of gastroesophageal reflux?* & $2.3(1.4)$ & $2.1(1.4)$ & 0.09 \\
\hline & Oncogenic potential of H. pylori & & & \\
\hline \multirow[t]{4}{*}{8} & What is the nature of the association between H. pylori and gastric cancer? & & & \\
\hline & Definite & $130(41.1)$ & $193(60.1)$ & $<0.001$ \\
\hline & Possible & $141(44.9)$ & $119(37.1)$ & \\
\hline & No connection & $18(5.7)$ & $4(1.3)$ & \\
\hline 9 & Do you agree that $H$. pylori eradication reduces the risk of developing gastric cancer ${ }^{* * ?}$ & $2.81(0.9)$ & $3.07(0.7)$ & $<0.001$ \\
\hline \multirow[t]{5}{*}{10} & How do you follow-up patients with gastric intestinal metaplasia?*** & & & \\
\hline & No need for surveillance & $10(3.5)$ & $8(2.5)$ & $<0.001$ \\
\hline & Recommend GI consult & $131(45.3)$ & $65(20.3)$ & \\
\hline & Recommend endoscopic surveillance & $199(68.9)$ & $244(76.0)$ & \\
\hline & Screening for H. pylori & & & \\
\hline 11 & Do you screen for $H$. pylori in first-degree relatives of gastric cancer patients?* & $1.4(1.56)$ & $2.4(1.6)$ & 0.07 \\
\hline 12 & Do you screen for H. pylori among asymptomatic family members?* & $0.7(1.1)$ & $0.7(1.1)$ & 0.98 \\
\hline 13 & $\begin{array}{l}\text { Do you screen for H. pylori prior to initiating long term therapy with non-steroidal anti-inflammatory } \\
\text { drugs?* }\end{array}$ & $0.9(1.3)$ & $1.0(1.2)$ & 0.47 \\
\hline 14 & $\begin{array}{l}\text { Do you screen for } H \text {. pylori prior to initiating long term therapy with non-steroidal anti-inflammatory } \\
\text { drugs in patients with a history of peptic ulcer disease?* }\end{array}$ & $\mathrm{n} / \mathrm{a}$ & $1.8(1.5)$ & na \\
\hline
\end{tabular}

*VAS 0-4 results expressed as mean (SD) where 0 , never; 1 , rarely; 2 , occasionally; 3 , usually; 4 , consistently

${ }^{* *}$ VAS 0-4 results expressed as mean (SD) where 0 , strongly disagree; 1 , disagree; 2 , equivocal; 3 , agree; 4 , strongly agree

${ }^{* * *}$ more than one answer accepted in 2015

Hp, Helicobacter pylori; PPIs, proton pump inhibitors; SD, standard deviation; PUD, peptic ulcer disease; GI, gastroenterology

\section{Predictors of compliance with $\boldsymbol{H}$. pylori treatment}

Variables that were significantly associated with compliance with first-line H. pylori treatment guidelines included a central clinic location (odds ratio [OR] 2.82, 95\% confidence interval [CI] 1.49-5.35; $\mathrm{P}=0.01$ ) and exposure to printed educational materials (OR 1.84, 95\%CI 1.16-2.91; $\mathrm{P}=0.01$ ) and the social media platform (OR 7.57, 95\%CI 3.70-15.48; $\mathrm{P}<0.001$ ). These 
factors remained significant following multivariate analysis (Table 4). Factors significantly associated with compliance with second-line treatment guidelines included female sex (OR 1.54, 95\%CI 0.97-2.44; $\mathrm{P}=0.02$ ), and lack of EOVs (OR 0.34, 95\%CI $0.14-0.85 ; \mathrm{P}=0.02)$. None of these factors remained significant following multivariate analysis.

\section{Discussion}

In our follow-up survey of PCPs, we found that, despite suboptimal compliance with the recommendation to treat H. pylori with 4 drugs (37.1\%), compliance has risen almost tenfold since 2015. Interventions such as distribution of educational printed material and electronic consultation over a social media platform may be effective in increasing the implementation of $H$. pylori treatment guidelines. Compliance with guidelines is superior among PCPs working in central, urban clinics, compared with those working in the rural periphery. This is the first study to assess changes in compliance with $H$. pylori treatment guidelines following a targeted intervention to educate PCPs.

Our finding that most PCPs do not account for a high degree of CLA resistance when prescribing first-line treatment for H. pylori infection is consistent with others [16,17]. A recent South American study found that over 95\% of PCPs prescribe standard TT despite the high rate of local resistance of $H$. pylori to CLA $(>35 \%)[18,19]$. A similar proportion of PCPs favoring TT has been reported in Italy, Turkey and South Korea [20-22]. On the other hand, a lower proportion of PCPs prescribed standard TT in Pakistan (61\%) and Mexico (64\%), and in our study $(63 \%)[23,24]$. The degree of CLA resistance in Israel is $>20 \%$ in adults; therefore, first-line treatment with TT should be abandoned [6,7]. According to the Maastricht V/Florence Consensus Report and the Toronto Consensus, treatment options include concomitant therapy with amoxicillin, CLA and a nitroimidazole, or BQT with bismuth, tetracycline, and a nitroimidazole, together with a proton pump inhibitor $[2,4]$. According to the American College of Gastroenterology's Clinical Guideline, sequential therapy with amoxicillin followed by CLA and a nitroimidazole, together with a proton pump inhibitor, remains a valid alternative [3]. We noted a significant increase in the number of PCPs who recommended treatment for $H$. pylori regardless of symptoms, and who recommended confirming eradication with a noninvasive test. This practice is consistent with the principles set forth in the Kyoto global consensus, in which $H$. pylori was characterized as an infectious disease [25]. H. pylori is considered an obligate pathogen and causes histological gastritis, which correlates poorly with symptoms. It follows, therefore, that treatment should be offered regardless of symptomatology and that eradication should be confirmed in all patients following treatment. Our results might suggest an increased acceptance of the Kyoto consensus.

We found that compliance with recommendations for second-line treatment was higher than compliance with recommendations for first-line treatment. After excluding PCPs who chose to refer patients to a gastroenterologist for second-line treatment, the proportion of PCPs who recommended guidelineappropriate second-line treatment was $92.5 \%$. This high baseline level of compliance may be the reason that exposure to printed materials and electronic consultation were not found to be significant predictors of compliance. Another reason for the lack of significance could be the fact that printed materials focused mainly on first-line, rather than second-line treatment.

Interestingly, we found that exposure to EOVs in the 3 months prior to completing the questionnaire was associated with lower compliance with second-line treatment recommendations. This, too, may be attributable to the high baseline adherence among respondents with and without EOV exposure (86.8\% and 95.1\%, respectively), or the fact that EOVs focused mainly on firstline treatment. Furthermore, there is a questionable correlation between EOVs within 3 months of participation and EOVs during the 3-year period during which our intervention was implemented. Another possibility is that EOVs unrelated to our study occurred (such as visits from industry representatives) and were recalled by study respondents.

We found that compliance with $H$. pylori screening prior to commencing long-term NSAID therapy is suboptimal, and that compared to 2015 these results are unchanged. A nonsignificant trend towards improvement in PCPs' screening of first-degree relatives of gastric cancer patients was observed, although rates remain inappropriately low (Visual Analogue Scale (VAS) 1.4 vs. 2.4 in 2015 vs. 2018, $\mathrm{P}=0.07$ ). Screening asymptomatic family members (which is unnecessary) remains uncommon and in accordance with guidelines (VAS 0.7 vs. 0.7 in 2015 vs. $2018, \mathrm{P}=0.98)$.

Limitations of our study include those inherent to any voluntary questionnaire, such as selection bias. PCPs mindful of continuing medical education are more likely to have completed the questionnaire. We could not reliably assess the frequency or distribution of EOVs during the three-year intervention period (much less that of the printed materials). For this reason we chose to assess EOVs and exposure to printed materials in the 3 months preceding the questionnaire as a surrogate. We could not presume that PCPs could reliably recall an encounter more than 3 months previously. The validity of this surrogate endpoint is unknown. Therefore our study lacks a true control group, not exposed to our interventions. Correlation between compliance with guidelines and exposure to EOVs and printed materials within the 3 months may not necessarily indicate a correlation between compliance with guidelines and exposure to our educational initiative, which took place over a period of 3 years. Another limitation is that we do not know how many of the PCPs who participated in the 2015 survey did so again in 2018. However, the impact of our intervention was measured on a representative sample of the entire PCP population, rather than on individual PCPs. Finally, we cannot be sure that the improvement in outcomes is entirely attributable to our intervention.

In conclusion, targeted education of PCPs seems to be an effective method to increase compliance with guidelines for the treatment of $H$. pylori. Internet and social media platforms may be the most effective means to enhance information-sharing between PCPs and gastroenterologists, and thereby enable a streamlined approach to $H$. pylori treatment. Nevertheless, 


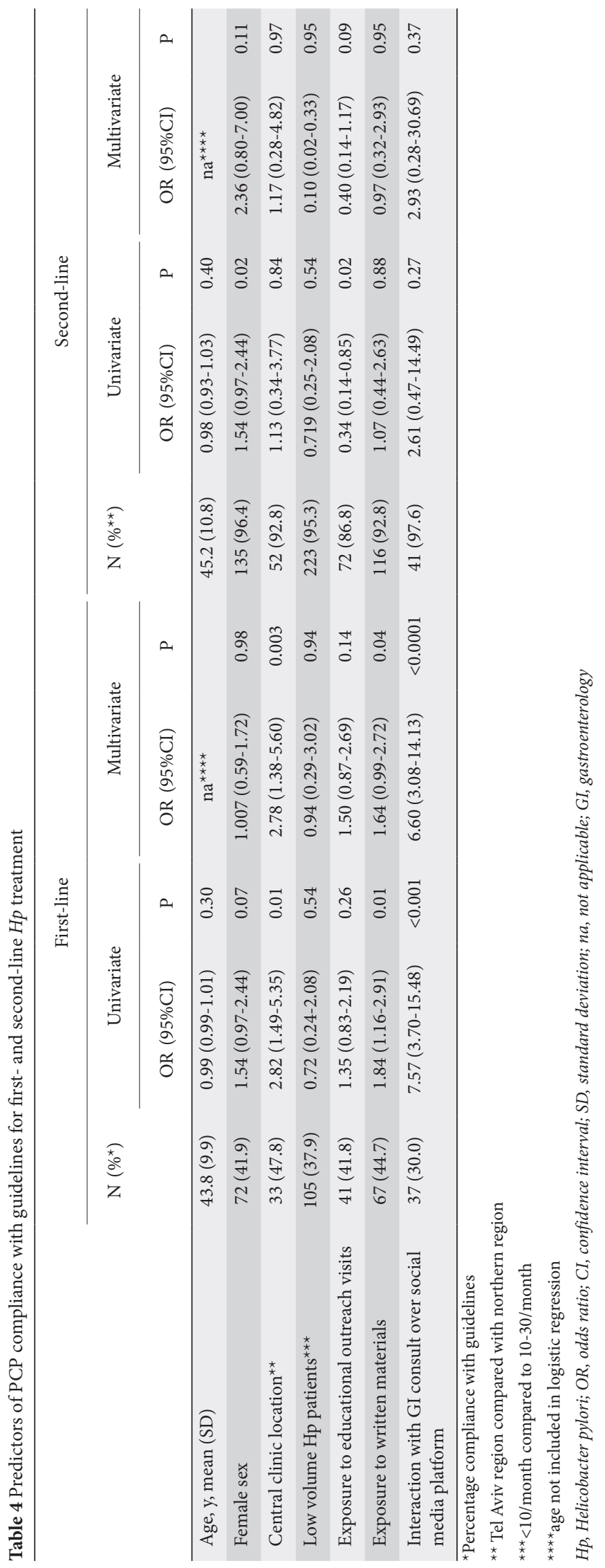




\section{Summary Box}

\section{What is already known:}

- Recent Helicobacter pylori (H. pylori) treatment guidelines recommend the use of 4 drugs for firstline treatment

- Adherence to H. pylori treatment guidelines is suboptimal

- Educational outreach visits and e-consultation have been shown to be effective means of changing primary care physicians' prescribing practices; however, they have not previously been assessed in the setting of $H$. pylori treatment

\section{What the new findings are:}

- Following a targeted intervention, compliance with $H$. pylori treatment guidelines increased almost 10-fold

- Variables significantly associated with compliance to first-line $H$. pylori treatment guidelines included a central clinic location (odds ratio $[\mathrm{OR}] 2.78$, 95\% confidence interval $[\mathrm{CI}]$ 1.38-5.60; $\mathrm{P}=0.003$ ), exposure to printed educational materials (OR 1.64, 95\%CI 0.99-2.72; $\mathrm{P}=0.04)$ and e-consultation over a social media platform (OR $6.60,95 \% \mathrm{CI}$ 3.08-14.13; $\mathrm{P}<0.0001$ )

- Internet and social media platforms seem to be the most effective means of enhancing information sharing between PCPs and gastroenterologists, and thereby enable a streamlined approach to $H$. pylori treatment

fewer than $40 \%$ of PCPs provide H. pylori treatment consistent with guideline recommendations. Therefore, steps should be taken to increase the penetration of the initiatives described.

\section{References}

1. Fock KM, Graham DY, Malfertheiner P. Helicobacter pylori research: historical insights and future directions. Nat Rev Gastroenterol Hepatol 2013;10:495-500.

2. Malfertheiner P, Megraud F, O'Morain CA, et al; European Helicobacter and Microbiota Study Group and Consensus panel. Management of Helicobacter pylori infection-the Maastricht V/ Florence Consensus Report. Gut 2017;66:6-30.

3. Chey WD, Leontiadis GI, Howden CW, Moss SF. ACG Clinical Guideline: treatment of Helicobacter pylori infection. Am J Gastroenterol 2017;112:212-239.

4. Fallone CA, Chiba N, van Zanten SV, et al. The Toronto Consensus for the treatment of Helicobacter pylori infection in adults. Gastroenterology 2016;151:51-69.

5. Boltin D, Kimchi N, Dickman R, Gingold-Belfer R, Niv Y, Birkenfeld S. Attitudes and practice related to Helicobacter pylori infection among primary care physicians. Eur J Gastroenterol Hepatol 2016;28:1035-1040.

6. Peretz A, Paritsky M, Brodsky D, Pastukh N, On A. Resistance of Helicobacter pylori to antibiotics in Israel: past vs present. Harefuah 2017;156:642-644.

7. Peretz A, Paritsky M, Nasser O, et al. Resistance of Helicobacter pylori to tetracycline, amoxicillin, clarithromycin and metronidazole in Israeli children and adults. J Antibiot (Tokyo) 2014;67:555-557.

8. O'Brien MA, Rogers S, Jamtvedt G, et al. Educational outreach visits: Effects on professional practice and health care outcomes. Cochrane Database Syst Rev 2007;4:CD000409.

9. Giguère A, Légaré F, Grimshaw J, et al. Printed educational materials: effects on professional practice and healthcare outcomes. Cochrane Database Syst Rev 2012;10:CD004398.

10. Vimalananda VG, Gupte G, Seraj SM, et al. Electronic consultations (e-consults) to improve access to specialty care: a systematic review and narrative synthesis. J Telemed Telecare 2015;21:323-330.

11. Gupte G, Vimalananda V, Simon SR, DeVito K, Clark J, Orlander JD. Disruptive innovation: implementation of electronic consultations in a Veterans Affairs health care system. JMIR Med Inform 2016;4:e6.

12. Hensel JM, Yang R, Rai M, Taylor VH. Optimizing electronic consultation between primary care providers and psychiatrists: mixed-methods study. J Med Internet Res 2018;20:e124.

13. Oltulu P, Mannan AASR, Gardner JM. Effective use of Twitter and Facebook in pathology practice. Hum Pathol 2018;73:128-143.

14. Rolls K, Hansen M, Jackson D, Elliott D. How health care professionals use social media to create virtual communities: an integrative review. J Med Internet Res 2016;18:e166.

15. Chan WS, Leung AY. Use of social network sites for communication among health professionals: systematic review. J Med Internet Res 2018;20:e117.

16. Fischbach W, Zerl A, Klassert C. How do primary care physicians manage their patients with Helicobacter pylori infection? Results of a survey and their implementation into the German S2k guideline 2016. Z Gastroenterol 2017;55:136-139.

17. Miehlke S, Loibl R, Meszaros S, Labenz J. Diagnostic and therapeutic management of Helicobacter pylori: a survey among German gastroenterologists in private practice. Z Gastroenterol 2016;54:1130-1137.

18. Escala Perez-Reyes AY, Jiménez Carranza AE, Bussalleu Rivera A. How do gastroenterologists in Peru deal with Helicobacter pylori infection? Study based on a survey conducted in 2014. Rev Gastroenterol Peru 2015;35:295-305.

19. Boehnke KF, Valdivieso M, Bussalleu A, et al. Antibiotic resistance among Helicobacter pylori clinical isolates in Lima, Peru. Infect Drug Resist 2017;10:85-90.

20. MacOni G, Tosetti C, Miroglio G, et al. Management of Helicobacter pylori-related gastrointestinal diseases by general practitioners in Italy. Aliment Pharmacol Ther 1999;13:1499-1504.

21. Canbaz S, Sunter AT, Peksen Y, Leblebicioglu H. Survey of general practitioners' knowledge about Helicobacter pylori infection. BMC Gastroenterol 2005;5:4.

22. Kim BG, Kim JW, Jeong JB, et al. Discrepancies between primary physician practice and treatment guidelines for Helicobacter pylori infection in Korea. World J Gastroenterol 2006;12:66-69.

23. Ahmed S, Salih M, Jafri W, Ali Shah H, Hamid S. Helicobacter pylori infection: approach of primary care physicians in a developing country. BMC Gastroenterol 2009;9:23.

24. Cano-Contreras AD, Rascón O, Amieva-Balmori M, et al. Approach, attitudes, and knowledge of general practitioners in relation to Helicobacter pylori is inadequate. There is much room for improvement! Rev Gastroenterol Mex 2018;83:16-24.

25. Sugano K, Tack J, Kuipers EJ, et al; faculty members of Kyoto Global Consensus Conference. Kyoto global consensus report on Helicobacter pylori gastritis. Gut 2015;64:1353-1367. 


\section{Supplementary Table 1}

\section{Study Questionnaire}

\section{Part A Treatment of Helicobacter pylori (H. pylori)}

Q1. What is your preferred first-line treatment for H. pylori infection?

1. 7-day clarithromycin-based triple therapy

2. 10-day clarithromycin-based triple therapy

3. 14-day clarithromycin-based triple therapy

4. Sequential therapy

5. Quadruple therapy (either bismuth or non-bismuth based)

6. Other (please specify)

Q2. What is your preferred second-line treatment for H. pylori infection?

1. Repeat the same treatment exactly

2. Use the same drugs as before but increase the dose and/or treatment duration

3. Referral to gastroenterologist for treatment

4. Metronidazole-based triple therapy

5. Bismuth-based quadruple therapy

6. Levofloxacin-based triple therapy

7. I do not attempt to treat $H$. pylori for a second time

\section{Part B Detection of H. pylori}

Q3. What is your preferred noninvasive test to diagnose H. pylori infection?

1. A validated IgG serological test

2. C13-urea breath test

3. Stool antigen test

4. I never test for H. pylori. If I suspect infection I refer to a gastroenterologist.

Q4. How many days do you withhold proton pump inhibitors and antibiotics prior to $H$. pylori testing?

1. 0 days

2. 5 days

3. 7 days

4. 14 days

5. 30 days

Q5. Do you confirm H. pylori eradication with a noninvasive test?

1. I routinely confirm eradication with a noninvasive test

2. I only confirm eradication in patients with ongoing symptoms

3. I only confirm eradication in patient with significant pathology, such as gastric ulcer or mucosa-associated lymphoid tissue lymphoma

4. I do not routinely confirm $H$. pylori eradication in any situation.
Q6. What do you do when confronted with a positive H. pylori test in an asymptomatic patient?

1. I treat all patients found to harbor $H$. pylori infection

2. I only treat symptomatic patients

3. I never treat for $H$. pylori infection

Q7. Do you seek H. pylori in patients with symptoms suggestive of gastroesophageal reflux?

1. H. pylori does not cause reflux symptoms; therefore, I do not seek the organism in this setting

2. Rarely

3. Occasionally

4. Usually

5. Consistently

\section{Part C Oncogenic potential of H. pylori}

Q8. What is the nature of the association between $H$. pylori and gastric cancer?

1. There is a definite link between $H$. pylori infection and gastric cancer. $H$. pylori may cause gastric cancer

2. The link between $H$. pylori infection and gastric cancer is equivocal

3. There is no connection between H. pylori infection and gastric cancer

Q9. Do you agree that $H$. pylori eradication reduces the risk of developing gastric cancer?

1. Strongly disagree

2. Disagree

3. Equivocal

4. Agree

5. Strongly agree

Q10. How do you follow-up patients with gastric intestinal metaplasia? (more than one response is permitted)

1. Intestinal metaplasia is commonly seen on pathology reports, has no clinical significance, and may be ignored.

2. I refer to a gastroenterologist for counseling

3. I refer for gastroscopy with biopsy every 2-3 years

\section{Part D Screening for $H$. pylori}

Q11. Do you screen for $H$. pylori in first-degree relatives of gastric cancer patients?

1. There is no recommendation to test healthy family members, therefore I do not seek the organism in this setting

2. Rarely

3. Occasionally

4. Usually

5. Consistently 
Q12. Do you screen for H. pylori among asymptomatic family members?

1. There is no recommendation to test healthy family members, therefore I do not seek the organism in this setting

2. Rarely

3. Occasionally

4. Usually

5. Consistently

Q13. Do you screen for $H$. pylori prior to initiating long-term therapy with non-steroidal anti-inflammatory drugs?

1. Never
2. Rarely

3. Occasionally

4. Usually

5. Consistently

Q14. Do you screen for $H$. pylori prior to initiating long-term therapy with non-steroidal anti-inflammatory drugs in patients with a history of peptic ulcer disease
6. Never
7. Rarely
8. Occasionally
9. Usually
10. Consistently 